\title{
Images Enhancement with Brightness Preserving using MRHRBFN
}

\author{
Narendra Singh Bagri \\ SolT, RGPV, Bhopal
}

\author{
Sanjeev Sharma \\ SolT, RGPV, Bhopal
}

\author{
Santosh Sahu \\ SolT, RGPV, Bhopal
}

\begin{abstract}
In the image processing, Images Enhancement with Brightness Preserving has many methods likes HE(Histogram Equalization), MHE(Multi-Histogram Equalization), IDBPHE(Image Dependent Brightness Preserving Histogram Equalization). We are proposed a novel methodology for the enhancement of images MRHRBFN (Multi-Resolution Histogram with Radial Bias Function Network). Enhancement process using pixel independent multi histogram method and Radial Bias Function Network. In process of our methodology image are decompose in terms of subbands. The sub band division perform by Curvelet transform. The Curvelet Transform divides two types of bands as higher band and lower band. The separation band of frequency generates a multiple matrix for input of radial bias Function Network. We have radial Bias function network work in low band data, because higher band data preserve brightness of image. The lower frequency matrix calculates bias and proceed weight factor when The lower value of frequency matrix regret reaches the mean value of given image. Finally we get better enhance image in comparison of multi histogram equalization.
\end{abstract}

\section{Keywords: H.E., M.H.E., I.D.B.P.H.E., M.R.H.R.B.F.N,} Curvelet Transform.

\section{INTRODUCTION}

Image processing refers to a set of computational techniques which accept images as input. All image processing techniques can be applied to frames. Image processing modifies pictures to improve them (enhancement, restoration), extract information (analysis, recognition), and change their structure (composition, image editing). Image processing using digital computers is the most common method because digital methods are fast, flexible, and precise.[5],[6].

The goal of image enhancement techniques is to improve a quality of an image such that enhanced image is better than the original image. In The process of Image Enhancement removing blurring and noise and increasing contrast. The original image might have areas of very high and very low intensity. In this case the mean intensity, contrast,and sharpness (amount of blur removal) could be adjusted based on the pixel intensity statistics in various areas of the image.[7],[8] Image enhancement techniques [5],[6] can be divided into two broad categories:

1. Spatial domain methods, which operate directly on pixels, and

2. 2. Frequency domain methods, which operate on the Fourier transform of an image.

Brightness preserving An effective method for brightness preserving is presented with a mapping function which is a mixture of global and local transformation function in the form radial Basis function that improve both the brightness and fine detail of the input image .This function enhance the image contrast and preserve brightness by starching the intensity level. .Experiment show that the proposed method compete well with the existing enhancement method [1],[13]. The rest of the paper is organized as follows. . In section 2 related works In section 3 Histogram; in section 4 the proposed method and The proposed algorithm. the experimental results based on the proposed method are displayed in section 5 .

\section{RELATED WORK}

$1 \mathrm{P}$. Rajavel, This paper proposes image-dependent brightness preserving histogram equalization (IDBPHE) technique to enhance image contrast while preserving image brightness. The curvelet transform and histogram matching technique are used to enhance image. The proposed IDBPHE technique undergoes two steps. (i) The curvelet transform is used to identify bright regions of the original image. (ii) Histogram of the original image is modified with respect to a histogram of the identified regions. Histogram of the original image is modified using a histogram of portion of the same image hence; it enhances image contrast while preserving image brightness without any undesired artifacts. A subjective assessment to compare the visual quality of the images is carried out. Absolute mean brightness error (AMBE) and peak signal to noise ratio (PSNR) are used to evaluate the effectiveness of the proposed method in the objective sense. The proposed method have been tested using several images and gives better visual quality and PSNR value as compared to several other methods[1].

2. Yu Wang, et.al, Histogram equalization is a simple and effective image enhancing technique. But in some conditions, the luminance of an image may be changed significantly after equalizing process; this is why it never be utilized in video system in the past. A novel histogram equalization technique, equal area dualistic sub-image histogram equalization, is put forward in this paper. First, the image is decomposed into two equal area sub-images based on its original probability density function. Then the two sub-images are equalized respectively. At last, we get the result after the processed sub-images are composed into one image. The simulation result indicates that the algorithm can not only enhance image information effectively but also keep the original image luminance well enough to make it possible to be used in video system directly.[2]

3. Soong-Der Chen et.al, Histogram equalization (HE) is widely used for contrast enhancement. However, it tends to change the brightness of an image and hence, not suitable for consumer electronic products, where preserving the original brightness is essential to avoid annoying artifacts. Bi-histogram equalization (BBHE) has been proposed and analyzed mathematically that it can preserve the original brightness to a certain extends. However, there are still cases that are not handled well by BBHE, as they require higher degree of 
preservation. This paper proposes a novel extension of BBHE referred to as Minimum Mean Brightness Error Bi-Histogram Equalization (MMBEBHE) to provide maximum brightness preservation. BBHE separates the input image's histogram into two based on input mean before equalizing them independently. This paper proposes to perform the separation based on the threshold level, which would yield minimum Absolute Mean Brightness Error (AMBE - the absolute difference between input and output mean). An efficient recursive integer-based computation for AMBE has been formulated to facilitate real time implementation. Simulation results using sample image which represent images with very low, very high and medium mean brightness show that the cases which are not handled well by HE, BBHE and Dualistic Sub Image Histogram Equalization (DSIHE), can be properly enhanced by MMBEBHE. Besides, MMBEBHE also demonstrate comparable performance with BBHE and DSIHE when come to use the sample images shown in [2] and [3],[25].

4. Abd. Rahman Ramli, et.al, Histogram equalization (HE) is widely used for contrast enhancement. However, it tends to change the brightness of an image and hence, not suitable for consumer electronic products, where preserving the original brightness is essential to avoid annoying artifacts. Bi-histogram equalization (BBHE) has been proposed and analyzed mathematically that it can preserve the original brightness to a certain extends. However, there are still cases that are not handled well by BBHE, as they require higher degree of preservation. This paper proposes a generalization of BBHE referred to as Recursive Mean-Separate Histogram Equalization (RMSHE) to provide not only better but also scalable brightness preservation. BBHE separates the input image's histogram into two based on its mean before equalizing them independently. While the separation is done only once in BBHE, this paper proposes to perform the separation recursively; separate each new histogram further based on their respective mean. It is analyzed mathematically that the output image's mean brightness will converge to the input image's mean brightness as the number of recursive mean separation increases. Besides, the recursive nature of RMSHE also allows scalable brightness preservation, which is very useful in consumer electronics. Simulation results show that the cases which are not handled well by HE, BBHE and Dualistic Sub Image Histogram Equalization (DSIHE), have been properly enhanced by RMSHE.[4]

\section{HISTOGRAM}

A histogram is one of the basic quality tools. It is used to graphically summarize and display the distribution and variation of a process data set. . A histogram consists of tabular frequencies. the frequency divided by the width of the interval. The total area of the histogram is equal to the number of data. The main purpose of a histogram is to clarify the presentation of data. In the most common form of histogram, the independent variable is plotted along the horizontal axis and the dependent variable is plotted along the vertical axis. The data appears as colored or shaded rectangles of variable area [21],[22].

\subsection{Histogram Equalization}

Histogram equalization is a method in image processing of contrast adjustment using the image's histogram. This method usually increases the global contrast of many images, especially when the usable data of the image is represented by close contrast values .[20]

\subsection{Multi Histogram Equalization}

Multi-HE, which consists of decomposing the input image into several sub-images, and then applying the classical HE process to each one. This methodology performs a less intensive image contrast enhancement, in a way that the output image presents a more natural look.[23],[24].

\subsection{IDBPHE}

The proposed image dependent brightness preserving histogram equalization (IDBPHE) technique use the wrapping discrete curve let transforms (WDCvT) and the histogram matching technique. A simple diagram of IDBPHE is shown in Fig. 3.3 and the corresponding steps are given below.

1. Region identification and separation: The curve let transform is used to identify bright regions of an original image.

2. Histogram computation and matching.

-A histogram of the original image and the histogram of pixels which belong to the identified regions are computed.

- Modify a histogram of the original image with respect to a histogram of the identified regions.[1],[10]

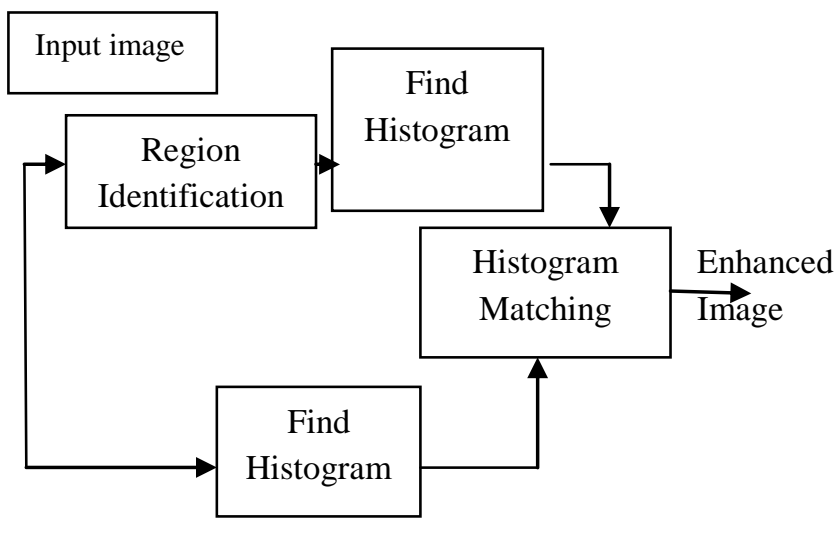

Fig3.3. Image enhancement

\section{PROPOSED METHOD}

Enhancement is a process for improving the appearance or stability for particular image and its applications.

To evaluate effectiveness of the proposed method absolute mean brightness error (AMBE) and peak signal to noise ratio (PSNR) are used. AMBE is used to assess the degree of brightness preservation. Smaller the AMBE is the better. Smaller AMBE indicates that mean values of the original and result images are almost same. PSNR is used to assess the degree of contrast enhancement. Greater the PSNR is the better[1]. Greater PSNR indicates better image quality. AMBE is given by,

$$
\operatorname{AMBE}(X . Y)=\left|M_{X}-M_{Y}\right|
$$

Where Mx, My represent mean values of the input image $X$ and output image $\mathrm{Y}$, respectively. PSNR is given by,

$$
\text { PSNR }=20 \log \left(\frac{\mathrm{I}_{\text {Max }}}{\sqrt{M S E}}\right)
$$

Mean squared error (MSE) is defined as,

$$
\operatorname{MSE}=\frac{1}{M N} \sum_{i=1}^{M} \sum_{j=1}^{N}\left|I(i, j)-I_{e}(i, j)\right|^{2}
$$


Where Imax is the maximum possible pixel value of the image, I, Ie are the original and enhanced images, respectively and $\mathrm{MxN}$ is size of the image. [1], [10].

\subsection{Multi Resolution Histogram with Radial Basis Function Networks (MRHRBFN)}

\subsubsection{Multi Resolution Histogram}

Multi histogram is a family of histogram obtained for multiple resolution of an image. Multi resolution histograms overcome the inability of a single histogram to encode the spatial feature of images.[7]. The multiresolution histogram shares many desirable properties with the plain histogram, including that they are both fast to compute, space efficient, invariant to rigid motions, and robust to noise.

The multiresolution histogram that uses the differences between histograms of consecutive image resolutions. We show that with our simple feature we achieve or exceed the performance obtained with more complicated features. Further, we show our algorithm to be the most efficient and robust.[11],[12].

\subsubsection{Radial Basis Function Networks}

A radial basis function network is a neural network approached by viewing the design as a curve-fitting (approximation) problem in a high dimensional space. Learning is equivalent to finding a multidimensional function that provides a best fit to the training data, with the criterion for "best fit". In a neural network, the hidden units form a set of "functions" that compose a random "basis" for the input patterns (vectors). These functions are called "radial basis functions". The design of a RBFN in its most basic form consists of three separate layers. The input layer is the set of source nodes (sensory units). The second layer is a hidden layer of high dimension. The output layer gives the response of the network to the activation patterns applied to the input layer. The transformation from the input space to the hidden-unit space is nonlinear. On the other hand, the transformation from the hidden space to the output space is linear. The dimension of the hidden-unit space high in an RBFN. [14]

$$
F(x)=\sum_{i=1}^{N} x_{i} \varnothing\left(|| x-x_{i}||\right)
$$

Where $\{\varnothing(|| x-x i||) / i=1,2, \ldots \ldots \mathrm{N}\}$, is a set of $\mathrm{N}$ random functions known as radial basis functions, and $\| .||$ represents a norm that is generally Eucliedian. The known data point $x_{i} \in R^{p}, \mathrm{i}=1,2, \ldots \mathrm{N}$ are the centers of radial basis function functions.

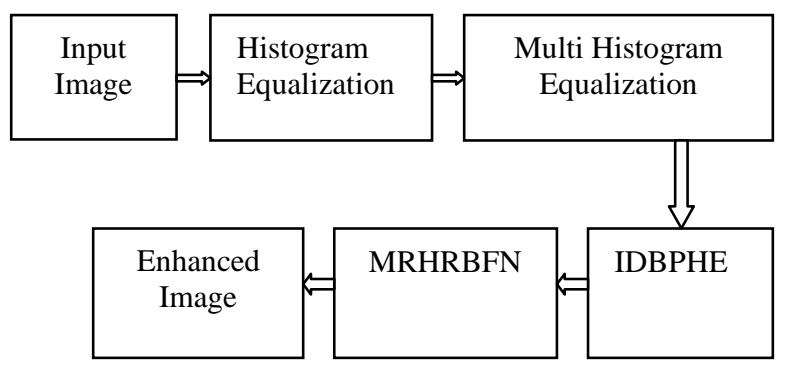

Fig 4: Block Diagram of Proposed Method
The flowchart of the Image enhancement algorithm is shown in Fig 4 The main steps of the proposed Method include:

\section{STEP1:}

Read the Input Image.

STEP2:

Find Histogram Equalization of Input Image.

STEP3:

Than Find Multi Histogram Equalization of Input Image.

\section{STEP4:}

Than we Perform IDBPHE (Image Dependent Brightness Preserving Histogram Equalization) Method for Region Identification and Separation. The curve let Transform is used to Identify Bright Region of an Original Image.

\section{STEP5:}

Finally we applied our Proposed Method MRHRBFN (Multi Resolution Histogram With Radial Basis Function Network) for Enhanced Input Image.

STEP6:

Finally we find Enhanced Image.

\subsection{Flow chart of proposed method}

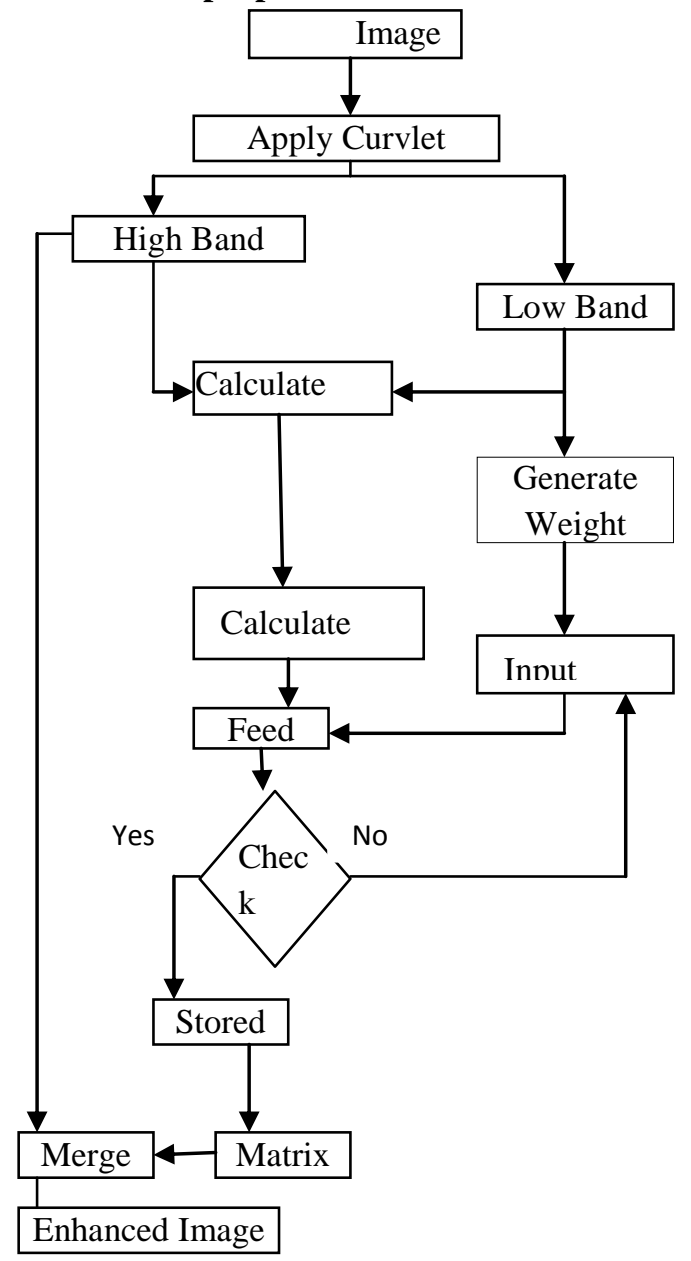

Fig. 4.1: Flow Chart of Our Proposed Method (MRHRBFN) 


\section{EXPERIMENTAL SETUP AND RESULTS}

In this section, a comparison among our proposed method is presented. A subjective assessment to compare the visual quality of the images is carried out. Absolute mean brightness error and peak signal noise ratio is used to assess the effectiveness of the proposed method. Former assess the degree of brightness preservation while later assess the degree of contrast enhancement.

The proposed method was tested with several gray and color images has been compared with different brightness preserving state-of-the-art histogram equalization methods HE[Histogram Equalization], MHE [Multi Histogram Equalization], IDBPHE [Image Dependent Brightness Preserving Histogram Equalization], MRHRBFN [Multi Resolution Histogram Equalization with radial Bias Function Network]

\subsection{For gray image}

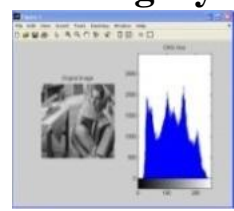

(a)

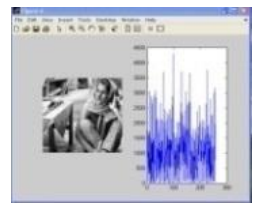

(d)

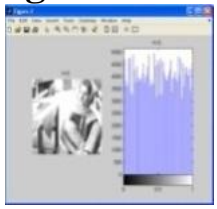

(b)

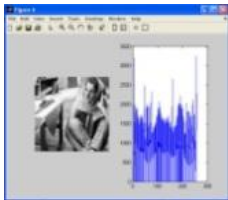

(e)

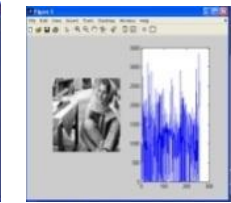

(c)
Fig 5.1: Histogram equalization results. (a) original image and enhanced output of (b) H.E, (c) M.H.E,(d)I.D.B.P.H.E , (e) M.R.H.R.B.F.N

Table 1 For gray image

\begin{tabular}{|l|l|c|}
\hline \multirow{2}{*}{ Methods } & \multicolumn{2}{|c|}{ Image } \\
\cline { 2 - 3 } & AMBE & PSNR \\
\hline H.E & 41.6821 & 11.9638 \\
\hline M.H.E & 40.2542 & 13.3809 \\
\hline I.D.B.P.H.E & 24.405 & 16.4803 \\
\hline M.R.H.R.B.F.N & 9.1341 & 27.533 \\
\hline
\end{tabular}

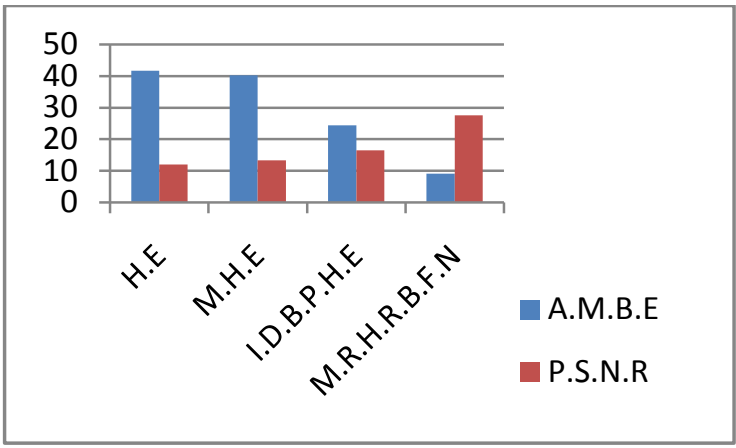

Chart 5.1: Result of different categories for gray images
5.2 For color image

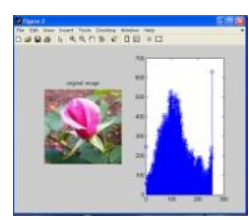

(a)

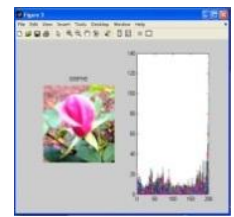

(d)

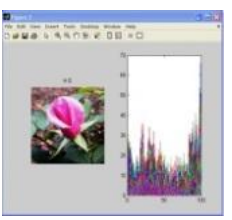

(b)

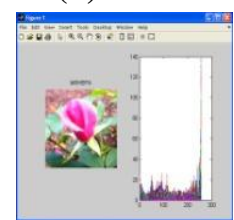

(e)

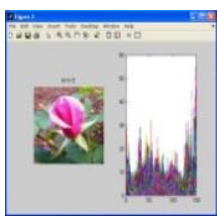

(c)
Fig 5.2. Histogram equalization results. (a) original image and enhanced output of (b) HE. (c) M.H.E. (d) I.D.B.P.H.E. (e) M.R.H.R.B.F.N.

\begin{tabular}{|l|l|l|}
\hline \multirow{2}{*}{ Methods } & \multicolumn{2}{|c|}{ Image } \\
\cline { 2 - 3 } & AMBE & PSNR \\
\hline H.E & 21.2638 & 6.7579 \\
\hline M.H.E & 25.7887 & 8.9351 \\
\hline I.D.B.P.H.E & 15.635 & 10.7394 \\
\hline M.R.H.R.B.F.N & 5.8518 & 17.639 \\
\hline
\end{tabular}

Table 2 For color image

For color image

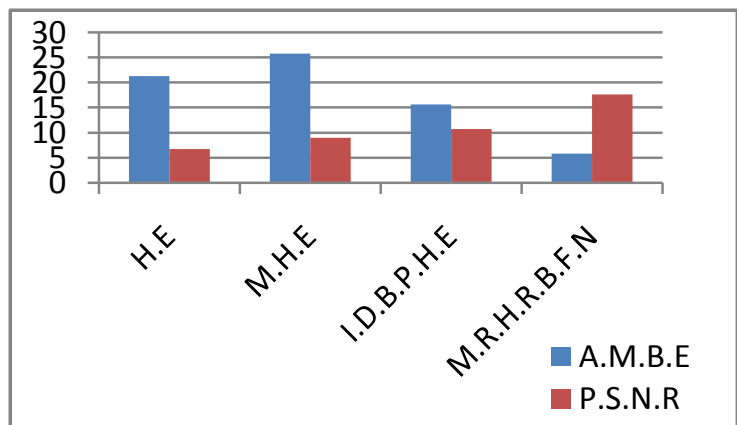

Chart 5.2: result of different categories for color images:

\section{CONCLUSION}

We proposed a Novel methodology for image enhancement based on pixel independency multi histogram resolution and radial bias function network in this method we decompose in terms of layers. Layers of image proceed as a input vector of neural network. Neural network updated the value on the bases of bias .bias supply the regenerated value of image matrix and get enhanced image. Our proposed method implements in mat lab software and take some standard image for comparison of result. The compare result is batter instead of previous method. Future work for our proposed methodology time complexity and process are simplified using radial bias function network. In this method shows the good performance for enhancement of gray and color Image. 


\section{REFERENCES}

[1] P.Rajavel "Image Dependent Brightness Preserving Histogram Equalization", IEEE Transactions on Consumer Electronics, Vol. 56, No. 2, May 2010.

[2] Yu Wan, Qian Chen and Bao-Min Zhang, "Image enhancement based on equal area dualistic sub-image histogram equalization method," IEEE Trans. Consumer Electron., vol. 45, no. 1, pp. 68-75,Feb. 1999.

[3] Yeong-Taeg Kim, "Contrast Enhancement Using Brightness Preserving Bi-Histogram Equalization," IEEE Trans Consumer Electronics, vol.43, no. 1, pp. 1-8, Feb. 1997.

[4] Soong-Der Chen and Abd. Rahman Ramli ,Member IEEE"Contrast enhancement using recursive meanseparate histogram equalization for scalable.

[5] Gonzalez C. and Woods E., Digital Image Processing, Addison-Wesley, 1992.

[6] A. Rafael C. Gonzalez, and Richard E. Woods, "Digital Image Processing," 2nd edition, Prentice Hall, 2002.

[6] Wang Y., Chen Q., and Zhang B., "Image Enhancement Based on Equal Area Dualistic Sub-Image Histogram Equalization Method," Computer Journal of IEEE Transactions on Consumer Electronics, vol. 45, no. 1, pp. $68-75,1999$.

[7] Andersen, Bjorn and Tom Fagerhaug. Root Cause Analysis; Simplified Tools and Techniques. Milwaukee, WI; ASQ Quality Press, 2000.

[8] William J. Mitchell. The Recon_gured Eye: Visual Truth in the Post-Photographic Era. MIT Press 1992.

[9] Andersen, Bjorn and Tom Fagerhaug. Root Cause Analysis; Simplified Tools and Techniques. Milwaukee, WI; ASQ Quality Press, 2000.

[10] Okes, Duke and Russ Westcott, eds. The Certified Qualiy Manager Handbook, Second Edition. Milwaukee, WI ASQ Quality Press, 2001. "Brightness Preserving Image Contrast Enhancement Using Weighted Mixture of Global and Local Transformation Functions.

[11] E. Hadjidemetriou, M. D. Grossberg, and S. K. Nayar Computer Science, Columbia University, New York, NY 10027," Multiresolution Histograms and their Use for Texture Classification"

[12] Qing Liu Yidong Yuan Xuemin Lin," Multi-resolution Algorithms for Building Spatial Histograms"

[13] Hasanul Kabir1, Abdullah Al-Wadud2, and Oksam Chae,"Brightness Preserving Image Contrast
Enhancement Using Weighted Mixture of Globaland Local Transformation Function",

[14] Adrian G.Bors,Department of computer Science university of York"Introduction of the Radial Basis Function (RBF) Networks".

[15] Rajesh Garg, Bhawna Mittal, Sheetal Garg "Histogram Equalization Techniques For Image Enhancement, Vol. 2 Issue 1, March 2011

[16] Hojat Yeganeh, Ali Ziaei, Amirhossein Rezaie," A Novel Approach for Contrast Enhancement Based on Histogram Equalization", Proceedings of the International Conference on Computer and Communication Engineering 2008, May 13-15, 2008 Kuala Lumpur, Malaysia

[17] Didar S. Sohi, S. S. Devgan,"Application to Enhance the Teaching and Understanding of Basic Image Processing Techniques".

[18] Wuni xu, Lanxiang Zhong, Dingyuan Wang"Image Processing Based on Seamless Integration Technology Between Lab VIEW and MATLAB. International Conference on Information, Networking and Automation (ICINA), 2010.

[19] Yafei TIAN, Qingtao WAN, Fengjun WU," Local Histogram Equalization Based on the Minimum Brightness Error", Fourth International Conference on Image and Graphics.

[20] H.D. Cheng * and X.J. Shi ,A simple and effective histogram equalization approach to image enhancement.

[21] Brassard, M. (1988). The Memory Jogger, A Pocket Guide of Tools for Continuous Improvement, pp. 36 - 43 Methuen, MA: GOAL/QPC.

[22] Department of the Navy (November 1992), Fundamentals of Total Quality Leadership (Instructor Guide), pp. 6-44 6-47. San Diego, CA: Navy Personnel Research and Development Center.

[23] Eric Wharton, Dr. Karen Panetta, Dr. Sos Agaian," human visual system based multi-histogram equalization for nonuniform illumination and shadow correction "

[24] D. Menotti, L. Najman, J. Facon and A. A. Araujo, "Multi-histogram equalization methods for contrast enhancement and brightness.

[25] Soong-Der Chen, Abd. Rahman Ramli, Member, IEEE "Minimum Mean Brightness Error Bi-Histogram Equalization in Contrast Enhancement", IEEE Transactions on Consumer Electronics, Vol. 49, No. 4, NOVEMBER 2003 